\title{
EDITORIAL
}

\section{COST OF SCALING}

Scaling has been a key driving force in the advancement of semiconductor technology. The economics is straightforward. For each generation, scaling shrinks the circuit dimension by $\sqrt{ } 2$, thus the transistor area by a factor of 2 . Design cleverness is supposed to shrink the transistor area by another factor of 2 to keep the circuit area constant for a quadruple increase of the number of transistors. If the processing cost is strictly area dependent, there is a $4 X$ increase of the number of circuits operating at a higher speed. With the reduced cost, there is a lot of room to reduce price per device per megahertz to entice the customers to move into the next generation. All the industries along the semiconductor food chain are aware of this. Tool and processing costs gain higher ceilings.

Of course, the reality is far from the ideal. First, design cleverness cannot be counted on for each generation. Therefore, there is generally an increase of chip size for each generation, the worst case being an increase of $\sqrt{2}$ at each side of the circuit. The cost is now doubled. That is still all right. The customers still enjoy a larger than $2 X$ gain in dollar/device/megahertz. However, the cost can become even higher due to the more difficult physics and more sophisticated tools required to support the scaling.

Let me use exposure tools as an example for price increases. In the I- $\mu \mathrm{m}$ era, IX full-wafer scanners were giving way to step-and-repeat aligners. The IBM San Jose factory bought one of the first GCA steppers. This was the first over-\$1-million tool IBM ever purchased. The price so impressed IBM executives that the chairman of the board flew in to admire it. Now, \$15-million tools are purchased in quantity. The price will quickly escalate to $\$ 25$ million, $\$ 50$ million, or even $\$ 100$ million perhaps, if the law of economy allows.

To support higher resolutions, the numerical aperture (NA) of the imaging lens of the exposure tool has to be increased. The cost of a lens is usually proportional to the tangent of the aperture angle whose sine defines the NA. Increasing NA also increases the size of the lens, making it more difficult to fabricate the lens and requiring more materials. The optical components of extreme ultraviolet (EUV) pose another type of cost increase. Because of the lack of transmissive material, all optical components have to be reflective. More than 40 pairs of interference layers have to be coated on 4 to 6 reflective surfaces of the condenser and 4 to 6 surfaces of the imaging lens. Most of the imaging optics use aspherical surfaces whose configurations have to be accurate to a fraction of the 13.4-nm wavelength. Needless to say, the surface finish has to be smooth with respect to the wavelength. All these translate to a higher cost for the exposure tool. At a certain point, it becomes uneconomical to increase the NA. One resorts to reducing the wavelength or moving to a higher level of resolution enhancement by reducing the $k_{1}$ factor defining lens resolution.

Wavelength reduction has its own reasons for cost increases. First, the lens materials have become more exotic, thus more expensive. Case in point, I-line lens materials are more expensive than G-line materials. The high-purity quartz used in deep-UV lithography is more expensive than I-line materials. $\mathrm{CaF}_{2}$ to supplement quartz in ArF lithography and as the sole material in $F_{2}$ lithography is more expensive than quartz. Moreover, the better quality required for a shorter wavelength often makes a given material more expensive, such as quartz and $\mathrm{CaF}_{2}$, from $\mathrm{KrF}$ to $\mathrm{ArF}$ and $\mathrm{ArF}$ to $\mathrm{F}_{2}$, respectively.

The price burden does not end here. $A$ new resist material using completely new platforms, more so with revolutionary chemistry, is costly to develop. Deep-UV materials with chemical amplification are more expensive than I-line resist materials. ArF resist materials are dramatically worse. They are easily three times more expensive than deep-UV resists. The materials for $F_{2}$ lithography, being rarer and more difficult to develop, are expected to be much more expensive than ArF resists.

The cost of new light sources has become a significant component in cost escalation. Initially, it was relatively inexpensive to switch from G- to I-line. One uses the same mercury arc lamp with incremental improvement for more output and better bandwidth in the desired portion of the spectrum. Switching to deep-UV called for a major change in light source. Even though a 254-nm line is available, the mercury arc lamp was no longer efficient for high-throughput and high-accuracy imaging. The KrF excimer laser had to be developed for the bandwidth and robustness required in a manufacturing operation. Needless to say, the light source has become much more expensive and its footprint has become a significant percentage of the total footprint of the exposure tool. Switching to $\mathrm{ArF}$ and $\mathrm{F}_{2}$ lasers to reduce the wavelength to 193 and $157 \mathrm{~nm}$, respectively, posed increasing difficulties in light source development, making it more expensive than ever.

Despite all these steep price increases, the cost explosion of light sources has not yet started. The current costly EUV light source can only support I wph throughput. The combined input power and conversion efficiency has to increase by 2 orders of magnitude to make the throughput comparable to current commercial exposure tools. We also foresee an enormous increase in operating costs when compared to the already burdensome excimer lasers.

Resolution enhancement techniques (RET) offer a way to save costs. To the first order, the same exposure tools and resist materials are used for the next generation. The cost increase should be minimal. However, if the RET 
utilizes phase-shifting masks, extra expense is called for. A special type of illumination calls for illumination setups that are usually expensive purchase options. Almost all forms of RET need optical proximity corrections (OPC). The OPC software, hardware, and running cost are becoming a significant part of mask cost, which is getting worse for each generation. When $k_{1}$ is reduced, the mask error factor inevitably increases, necessitating tighter mask $C D$ and placement specifications that lead to an even higher cost.

In brief, material costs can increase as much as $3 X$ per generation, and mask costs also about $3 X$. The exposure tool cost has increased by $2 \mathrm{X}$ from $\mathrm{KrF}$ to $\mathrm{ArF}$ and is projected to be $3 \mathrm{X}$ from $\mathrm{KrF}$ to $\mathrm{F}_{2}$, possibly 5 to $10 \mathrm{X}$ from KrF to EUV. Who can afford them? In the early eighties, I was selected to give my view on the future of lithography to the IBM chief scientist and the Science Advisory Board. Several conclusions were made. First, optical lithography will be extended to its full potential by
NA increases and wavelength and $k_{1}$ reductions, beyond what any other types of lithography can do in a manufacturing environment. Second, the law of economics will prevail in setting the limit of lithography instead of the law of physics. This is indeed happening.

While we are pushing the forefront of the technology, let us not put any less emphasis on economy. Happy reading! Hard working! Pennypinching!

\section{Burn J. Lin Editor-in-Chief}

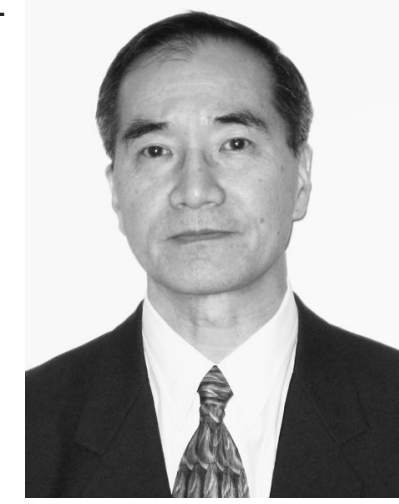

